\title{
Tres elementos sinérgicos para aprendizajes en línea
}

\author{
Laura Regil Vargas', Jaime Sabines Córdova² \\ ${ }^{1}$ Área Académica: Aprendizajes en Ciencias, Humanidades y Artes. Universidad \\ Pedagógica Nacional. - México \\ ${ }^{2}$ Sistema de Universidad Abierta y Educación a Distancia - Facultad de Ciencias \\ Políticas y Sociales - Universidad Nacional Autónoma de México - México \\ Iregil@upn.mx, jsabines_70@comunidad.unam.mx \\ Resumen. El objetivo de este artículo es reflexionar sobre el proceso de \\ aprendizaje en el aprendizaje en línea, a través del análisis de tres elementos \\ determinantes: Recursos Educativos Abiertos (REA), Diseño de interacción, \\ interactividad o navegación (IxD) y Experiencia de Usuario (UX), ya que la \\ articulación ponderada de estos tres elementos puede crear un vínculo sinérgico \\ que favorece la construcción de aprendizajes significativos. Reconocemos los \\ REA como el elemento principal, nos interesa conocer sobre el papel que juegan \\ los otros dos elementos. La propuesta metodológica: conocer el todo por sus \\ partes; desagregar los tres elementos para observarlos aisladamente y luego, \\ examinar el vínculo sinérgico al articularse.
}

Abstract. The purpose of this article is to reflect on the learning process in eLearning, through the analysis of three crucial elements: Open Educational Resources (OER), interaction design, navigation and interactivity (IxD) and User Experience $(U X)$ as a weighted linkage of these elements may create a synergistic link favors the construction significant learning. We recognize the $R E A$ as the main element, we are interested to know about the role played by the other two elements. The proposed methodology: to know the whole by its parts; disaggregating the three elements to observe them individually and then examine the synergistic link to be articulated.

Resumo. O objetivo deste artigo é refletir sobre o processo de aprendizagem na aprendizagem on-line, através da análise de três elementos cruciais: Recursos Educacionais Abertos (REA), Design de interação, de navegação $e$ interatividade (IXD) e Experiência do Usuário (UX) como a ligação ponderada destes elementos podem criar uma ligação sinérgica favorece a construção de aprendizagem significativa. Reconhecemos a REA, como elemento principal, estamos interessados em saber sobre o papel dos outros dois elementos. A metodologia proposta: satisfazer o conjunto das suas partes; desagregar os três elementos para observar o isolamento e, em seguida, examinar a relação sinérgica de articular. 
CBIE-LACLO 2015

Anais dos Workshops do IV Congresso Brasileiro de Informática na Educação (CBIE 2015)

\section{Introducción}

La temática que se desarrolla en este artículo está fundamentada en la siguiente hipótesis: Los Recursos Educativos Abiertos (REA) son un elemento importante en el proceso de construcción de aprendizajes significativos en tanto amplían la estructura cognoscitiva de los estudiantes en la comprensión de temas específicos y ayudan a resolver intereses y necesidades académicas particulares; además, porque la interacción con diversos recursos didácticos favorece la articulación de saberes, así el estudiante relaciona nuevos contenidos con sus conocimientos previos (Ausubel, 1983).

Para que este supuesto se cumpla, el diseño de los REA debe apoyarse en las siguientes condiciones: guardar coherencia con todos los elementos sustanciales al proceso educativo, integrarse a un diseño general de estructura de interacción, estar disponibles en soportes tecnológicos convenientes, ser mediados por docentes y, en consecuencia, generar experiencias productivas.

Como sabemos, por sí mismos no mejoran los procesos de aprendizaje. Sin embargo, reconocemos que los REA incentivan aproximaciones a los contenidos educativos, así como a otros dispositivos, temáticas y recursos tecnológicos y educativos.

Tener como punto de partida esa hipótesis nos lleva a presentar aquí la categorización de los elementos de nuestro supuesto y el análisis de la relación que guardan los REA con los elementos del diseño de interactividad (IxD) y con la experiencia del usuario (UX).

Pretendemos visualizar el diseño de la interactividad como un componente que permite la participación activa entre los usuarios y de éstos con el hardware y software, que facilita el acceso y los recorridos dentro del sistema, y que evidencia la esencia misma de la interactividad que radica en la conversación bidireccional receptor-emisor (Regil, 2003). Diálogo que se replica no sólo entre los usuarios sino con el mundo virtual, generando con ello una nueva percepción de los contenidos educativos, integrados en los REA; es decir, una experiencia de usuario asociativa, derivada de la intencionalidad de búsqueda y de rastreo, y comprensión de información especializada.

Fundamentalmente nos guía el objetivo de analizar el vínculo sinérgico entre tres elementos fundamentales de la educación en línea: los REA, el diseño de la interactividad y la experiencia de usuario, para descifrar si en ese vínculo se genera un efecto de sinergia que fortalece los aprendizajes significativos. La siguiente figura sintetiza gráficamente esta idea. 
CBIE-LACLO 2015

Anais dos Workshops do IV Congresso Brasileiro de Informática na Educação (CBIE 2015))

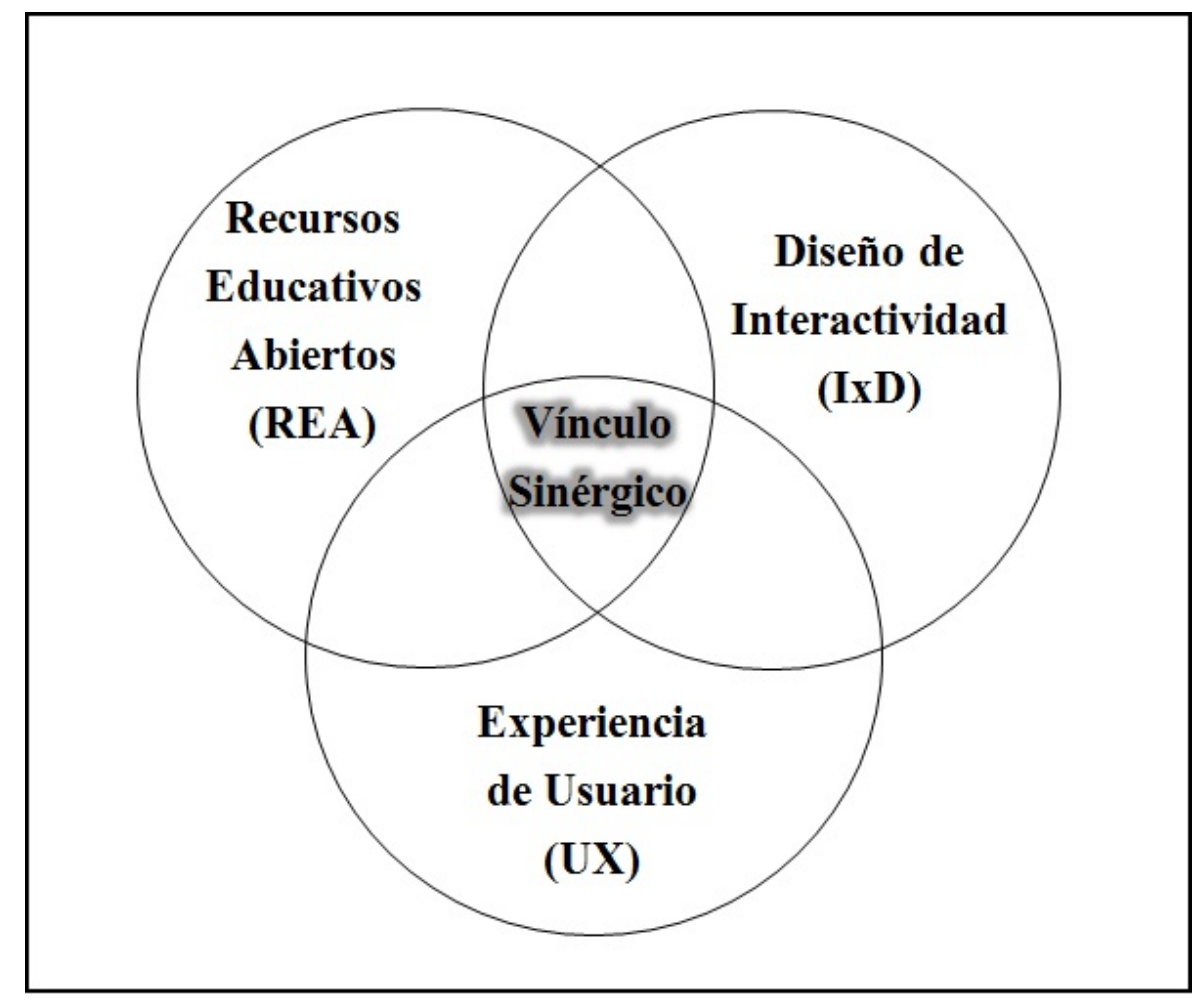

Figura 1. Vínculo Sinérgico

Se compone de cuatro apartados; en los tres primeros se sistematiza la descripción de los tres elementos desde la perspectiva de la educación en línea y en el último apartado exponemos la propuesta esencial del artículo, es decir, el análisis del vínculo sinérgico, una invitación al lector a observar la correlación ponderada de los tres elementos en favor de los aprendizajes significativos. En el primer apartado presentamos la fundamentación teórica de los REA así como algunas de sus características; el segundo, plantea la asociación del usuario con las herramientas, instrumentos y contenidos educativos en entornos virtuales, es decir, expone el diseño de la interacción; y el tercero registra la importancia de la experiencia del usuario de la educación en línea derivada de su contacto con los REA.

Este artículo, vehículo de divulgación de la sistematización y el análisis, contribuye a visibilizar estrategias e iniciativas, busca profundizar en los tópicos destacados en el Workshop Recursos Educacionais Abertos y pretende propiciar el intercambio entre profesionales. Será de utilidad para docentes universitarios, investigadores y analistas, así como para los alumnos involucrados en la modalidad de educación en línea; también interesará a informáticos, bibliotecarios y, a todos los profesionales vinculados a la educación virtual.

\section{Recursos Educativos Abiertos}

A partir de las recomendaciones realizadas en el año 2012 por la Organización de las Naciones Unidas para la Educación, la Ciencia y la Cultura (UNESCO por sus siglas en inglés) que proponen -entre otras encomiendas- fomentar el conocimiento y uso de los 
CBIE-LACLO 2015

Anais dos Workshops do IV Congresso Brasileiro de Informática na Educação (CBIE 2015)

REA, diversas instituciones de educación superior en el mundo han construido el andamiaje para alcanzar los objetivos educativos con apoyo en los REA.

De acuerdo con algunas investigaciones los REA son un apoyo en el proceso educativo y contribuyen en la construcción de aprendizajes significativos (Mortera, Ramírez y Burgos, 2011; Fini, 2009; Mora, 2008; Ramírez y Mortera, 2009; y Glasserman y Ramírez, 2011). Estos estudios revelan su eficacia en la integración de proyectos educativos, asimismo constatan su naturaleza como elementos constituyentes de conocimiento, al compartir en la red digital contenidos educativos en espacios virtuales; confirman así que el uso de REA conlleva a una reconceptualización de la forma de aprender y enseñar, y sirven como base para la apropiación de nuevos conocimientos que reorganizan la estructura cognitiva de los usuarios, favoreciendo así el proceso de aprendizaje.

En educación en línea los REA están “destinados a eliminar las barreras espaciotemporales entre las personas, y a potenciar los escenarios y entornos interactivos” (Martínez Guzmán, 2013: 241). Desde esta perspectiva, su uso en programas curriculares específicos permite aprovechar características propias como la accesibilidad y el potencial de exposición al conocimiento colectivo. Son un elemento fundamental en la educación en línea porque ayudan a mostrar o redescubrir contenidos disciplinares de forma integral, es decir, al identificar y seleccionar los REA que enriquecen los contenidos inherentes a una unidad de aprendizaje deseada y bajo una perspectiva extendida o diferente, el usuario incrementa sus saberes, que de forma tradicional sería más difícil de lograr.

En la actualidad, la creación de REA es una práctica común y progresiva derivada, no sólo de la propuesta de la UNESCO sino del uso de las tecnologías de la información y la comunicación; estos recursos forman parte de los resultados de diversas investigaciones así como del "movimiento opensource”, de los años noventa (Warger, 2002; DiBona, 1999). Los REA se han desarrollado con el fin de estimular y promover de manera estratégica el desarrollo del proceso educativo, componen una extensa gama de colecciones, como software libre, repositorios, objetos de aprendizaje, contenidos textuales o multimedia, herramientas digitales y entre otros, aplicaciones informáticas; que sin duda, favorecen la construcción de aprendizajes significativos.

\section{Diseño de la interactividad}

Los avances tecnológicos de los lenguajes de programación han revolucionado el diseño de la interactividad (IxD). Aunque éste pareciera un tema circunscrito al ámbito meramente técnico, importa en el análisis de los ambientes virtuales de aprendizaje, especialmente por el papel que juega en involucrar al usuario con los REA. De ahí que en el análisis de los elementos que intervienen en los modelos de educación a distancia éste concierne a todos los profesionales implicados, así como -indirectamente- a los estudiantes.

No subestimar sus alcances ni relegarlos al ámbito de lo técnico nos ayuda a registrar las diferentes dimensiones en las que el IxD involucra al usuario con los contenidos. El acoplamiento de sus componentes es guiado por su objetivo central: invitar y guiar la interactividad del usuario. Esto sucede en varias dimensiones; inicialmente, dispone la ubicación de las vías de acceso; luego, genera una gama de posibles recorridos 
a través de los contenidos; y por último, puede determinar diferentes formas de exploración. Es decir, el IxD debe estar ideado para provocar determinada interactividad, ya que, ciertos descuidos en su creación, pueden obstaculizar y producir desvíos e inclusive, desorientaciones.

En entornos virtuales la interactividad busca lograr que se cumplan los procesos de comunicación y de enseñanza-aprendizaje entre el docente y el alumno a través de la exposición de los contenidos disciplinares, con el uso de la infraestructura tecnológica. Sabemos que "la eficacia de la interfaz radica en su capacidad para implicar o no al usuario en el programa [...] para propiciar la interactividad. Por lo tanto debe ser preciso, homogéneo, atractivo y sugerente” (Regil, 2003). Para ello el IxD se basa en estudios sobre la percepción del usuario promedio y sobre pronósticos de exploración de los contenidos digitales; incluye aspectos como arquitectura de la información, estrategia de contenidos, diseño de la interfaz y diseño visual.

En ese sentido, es recomendable que el equipo de expertos encargados del desarrollo de ambientes virtuales de aprendizaje tenga presentes criterios como: claridad, sencillez, precisión y velocidad de respuesta. Ya que el usuario promedio puede explorar los contenidos digitales cuando el diseño de la interfaz se fundamenta en los principios básicos del IxD, es decir, cuando puede: ubicar, situar, visualizar, gestionar, recorrer y asociar los contenidos.

Desde esa perspectiva, para cerrar este apartado, afirmar que un eficiente IxD genera efectos sinérgicos entre el usuario y los contenidos del entorno virtual, ya que facilita la interactividad y apoya al usuario en la asociación de elementos. En ambientes virtuales de aprendizaje, un IxD orientado a la promoción de construcción de aprendizaje puede dinamizar los REA.

\section{Experiencia de usuario}

Séneca afirmó “Ningún viento es favorable para quien no sabe a dónde va”. En ese mismo sentido podemos afirmar que no hay experiencia de usuario (UX) que sea verdaderamente productiva si no se tienen claros los objetivos de la navegación y de exploración de los contenidos. Igual sucede para el estudiante que, en un ambiente virtual, no es consciente de sus propias metas académicas.

La idea de la UX en paralelo al “viento” de Séneca se basa en algunas de las ideas sobre las recientes teorizaciones sobre la "economía de la experiencia” (Pine \& Gilmore, 1999; Boswijk, Thijssen \& Peelen, 2007; Nijs, 2003). Epistemológicamente, éste es un constructo axiomático, por tanto es recomendable cierta mesura en la adopción de sus principios generales, ya que todavía guarda más relación con la mercadotecnia, en donde lo trivial y superficial suele presentarse como lo más atractivo. No obstante, para el tema que nos ocupa existen aportaciones importantes (Boswijk, Thijssen \& Peelen, 2007; Nijs, 2003). Por ejemplo, reseñas en donde se documentan tendencias como: búsqueda de experiencias para expresarse, conocer, aprender, compartir, y conservar recuerdos de esa situación. Subrayamos el último aspecto para insistir en la importancia de lo memorable o recordable de la experiencia.

La UX responde a los elementos que orientan al usuario, a la realización de recorridos productivos en la plataforma. Se fundamenta en su sensación de control e 
independencia respecto a su propio ritmo de autorregulación y en la toma de decisiones y de acciones (Nielsen, 1994 y 2003; Rodríguez, 2014). La mayoría de los usuarios pretende satisfacer intereses y necesidades específicas al navegar en la red digital; el proceso para conseguirlo genera un conjunto de sensaciones que llamamos "experiencia"; es decir, situaciones intangibles pero no imperceptibles. Por lo general los ritmos, los intereses y las necesidades de los estudiantes inscritos en un programa a distancia, son más definidos, tiene mayor grado de particularidad y se orientan a asignaciones temáticas especiales. En esos casos, en términos académicos, la UX resulta más demandante.

En este sentido, conviene apoyarse en algunos de los principios que Nielsen (2003) establece para la usabilidad y que consideramos útiles en la generación de una UX académicamente productiva.

- Simplificar la localización de información y la identificación de rutas de navegación.

- Facilitar aprendizajes comprobables

- Provocar sensación de logro y satisfacción

- Hacer que la experiencia sea compartible y memorable

Con estos argumentos invitamos a valorar la importancia que adquiere la UX como un elemento por atender en el diseño de REA y del IxD.

\section{Vínculo sinérgico de los tres elementos}

Iniciamos este artículo con la hipótesis que nos ha guiado hasta este apartado, punto medular del artículo: la articulación ponderada de los tres elementos, descritos en los apartados anteriores. Establecimos entonces que la construcción de aprendizajes significativos se apoya en el uso estratégico de los REA, los cuales pueden estar determinados por el binomio: IxD y UX. El análisis hasta aquí presentado nos lleva a afirmar que cuando el diseño de los REA atiende los principios del IxD es posible lograr una UX de aprendizaje que trasciende en construcción de conocimientos.

En educación los efectos sinérgicos se orientan hacia la promoción de los aprendizajes; específicamente buscan estimular logros que originen la continua dinamización de los procesos reflexivos. En educación en línea este tipo de desafíos se concentran en la estratégica articulación de recursos y contenidos en un espacio virtual. Por ejemplo, investigaciones recientes (Regil, 2014) sostienen que para algunos estudiantes universitarios la UX no siempre resulta plena, ya que algunos IxD retan a su intuición y desafían su paciencia. Esto puede entorpecer los trayectos en la exploración y hacer que no se logren los objetivos académicos. Es decir, ponderar uno de los elementos sin considerar o valorar suficientemente los otros dos conlleva desajustes que suelen desembocar en medianos resultados. Además, recordar que cada objetivo educativo implica articulaciones diferenciadas.

Por fortuna existe una amplia variedad de ejemplos de REA en donde se ha conseguido un efecto sinérgico, gracias a que se han ponderado los ingredientes del IxD y, como resultado se logra una UX significativa en cuanto a aprendizajes. Por ejemplo: la "Red Universitaria de Aprendizaje - UNAM" (RUA); es un portal que estimula y dirige al usuario (profesor/alumno) a experimentar interactivamente con sus contenidos, mismos que van desde medios multimedia, textos disciplinares, vínculos externos asociados a la 
temática, material de autoevaluación, recursos interactivos y videos, por mencionar algunos; el sitio web requiere para acceder a ciertos recursos, el registro (gratuito) del usuario. Uno de los objetivos del portal es proporcionar -a través de una interfaz atractiva y de fácil uso- la integración de contenidos disciplinares y REA de algunos de los programas de asignatura de los niveles educativos de bachillerato y licenciatura de la propia institución, recursos generados por miembros de la comunidad UNAM y de otras instituciones educativas en México, acordes con las normas nacionales e internacionales en materia de propiedad intelectual.

En síntesis, la sinergia se logra a través del ensamblado estratégico de los tres elementos. Mejorar el diseño de los REA contribuye para que el usuario-estudiante pueda aprender contenidos específicos y se posibilite la interactividad que le permita asociar elementos de manera eficaz. Ambas acciones no logran sus objetivos si no se es capaz de anticipar las sensaciones, logros y frustraciones que el espacio virtual genera. Por lo tanto, la generación del vínculo sinérgico aludido es el resultado de acciones concretas de un equipo transdisciplinar, en donde la conjunción de saberes y habilidades conduzcan a la integración de los tres elementos.

\section{Conclusiones}

Las perspectivas tradicionales sobre el uso de materiales educativos y particularmente sobre el proceso de enseñanza-aprendizaje, se ven rebasadas con la implementación de REA adaptados a los ambientes virtuales de aprendizaje; espacios que demandan no solamente un nuevo estilo de enseñar y aprender, sino una forma dinámica de presentación de contenidos y de comunicación eficaz. Por una parte, los instrumentos o herramientas así como los elementos constitutivos del proceso de enseñanza-aprendizaje utilizados en la educación en línea, deben generar un espacio de comunicación e interacción adecuado entre los docentes y los alumnos, y con ello generar una experiencia de usuario productiva.

El desarrollo de habilidades tecnológicas y el manejo de los contenidos educativos son algunos de los desafíos que este tipo de usuario enfrenta cotidianamente para desenvolverse en la cultura digital. Nuevas destrezas que demandan dominar al menos uno de los pilares de la educación, señalados por Delors (1994) en su obra colectiva, el “aprender a conocer". En ambientes virtuales, se trata de un saber concatenado con la interacción que el usuario experimenta en dichos espacios. Aprender a conocer implica no sólo la transmisión de conocimientos sino el comprenderlos, conocerlos y redescubrirlos; en proyectos curriculares formativos son precisamente los REA los que pueden apoyar este proceso.

En la relación tecnología y educación es conveniente no afiliarse a las novedades, sin antes haber analizado su verdadero potencial didáctico; con todo lo cautivador que suele ser lo novedoso. En educación en línea se busca lograr una sencilla articulación de los elementos, que se traduzca en una sólida certeza para los usuarios y en donde la precisión se alía con la sencillez para hacer factibles recorridos eficaces. La reducción de la curva de aprendizaje suele apoyarse en este tipo de coartadas.

En algunos modelos educativos hemos visto desvanecerse las formas escrupulosas de lo académico para dar lugar a la trivialidad de la mercadotecnia que envuelve a la 
CBIE-LACLO 2015

Anais dos Workshops do IV Congresso Brasileiro de Informática na Educação (CBIE 2015)

tecnología. En cambio, cuando la creatividad, el diseño y la simplificación de la tecnología potencian lo académico, se evidencian los logros en educación.

Por último, señalar que la integración de los REA en ambientes educativos en línea y particularmente en las prácticas docentes, se verá potenciada en la medida que estén disponibles, se difundan, sean alcanzados por los usuarios mejorando su interacción, y generen una experiencia de usuario efectiva, eficiente y satisfactoria. El vínculo sinérgico a que alude este artículo no se forma por generación espontánea; se trabaja desde las trincheras académicas.

\section{Referencias}

Ausubel, D. (1983). Teoría del aprendizaje significativo. Recuperado el 27 de junio de 2015, de http://bit.ly/SnBgCK.

Boswijk, A., Thijssen, T., \& Peelen, E. (2007). The experience economy: A new perspective. Pearson Education.

Delors, Jacques (1994). Los cuatro pilares de la educación. En La Educación encierra un tesoro. México: El Correo de la UNESCO, pp. 91-103. Recuperado el 28 de junio de 2015, de http://bit.ly/1eWCO9D.

DiBona, Chris \& Ockman, Sam (1999). Open Sources: Voices from the Open Source Revolution. Recuperado el 25 de junio de 2015, de http://oreil.ly/1JmnW9w.

Fini, A. (2009). The Technological Dimension of a Massive Open Online Course: The case of CCK08 Course Tools. International Review of Research in Open and Distance Learning, 10 (5), 1-26. Recuperado el 28 de junio de 2015, de http://bit.ly/1qrbD28.

Glasserman, L. D. y Ramírez, M. S. (2011). Generación de recursos educativos abiertos y móviles para la formación de investigadores educativos: estudio de casos a través de un esfuerzo interinstitucional. Memorias del XI Congreso Nacional de Investigación Educativa. Monterrey, Nuevo León, México. Recuperado el 28 de junio de 2015, de http://bit.ly/1JgVQtH.

Martínez Guzmán, María Dolores et all (2013). Los Recursos Educativos Abiertos para la Formación en el Trabajo. En Revista científica electrónica de Educación y Comunicación en la Sociedad del Conocimiento Publicación en línea (Semestral) Granada (España) Época II Año XIII Número 13 Vol. II Julio-Diciembre de 2013 ISSN: 1695-324X. Recuperado el 20 de junio de 2015, de http://bit.ly/1FtRXwg.

Mora, M. J. (2008) Open educational resources: motivations, governance, and content protection (Tesis de maestría) Carleton University, Ottawa, Canada. Recuperado el 28 de junio de 2015, de http://bit.ly/1Kj4t8i.

Mortera, F. J., Ramírez, M. S. y Burgos, J. V. (2011, junio). Vinculando Repositorios Digitales Educativos y Construyendo Comunidades de Práctica: Avances del Proyecto del Metaconector de Repositorios del CUDI-CONACYT. Ponencia presentada en el XII Encuentro de Virtual Educa, Distrito Federal, México. Recuperado el 28 de junio de 2015, de http://bit.ly/1TYbJLi.

Nielsen, J. (1994). Usability engineering. Massachusetts: AP Profesional. 
CBIE-LACLO 2015

Anais dos Workshops do IV Congresso Brasileiro de Informática na Educação (CBIE 2015)

Nielsen, J. (2003). Designing web usability: The practice of simplicity. Thousand Oaks: New Riders Publishing.

Nijs, D. (2003). Imagineering: Engineering for imagination in the Emotion Economy. Creating a fascinating world. Breda University of Professional Education, 15-32, Breda.

Organización de las Naciones Unidas para la Educación, la Ciencia y la Cultura-UNESCO (2002). Forum on the Impact of Open Courseware for Higher Education in Developing Countries Final report. Recuperado el 20 de junio de 2015, de http://bit.ly/1GtB8lZ.

Organización de las Naciones Unidas para la Educación, la Ciencia y la Cultura-UNESCO (2012). Declaración de París de 2012 sobre los REA. Recuperado el 20 de junio de 2015, de http://bit.ly/1E8xyz6.

Pine, B.J. y J.H. Gilmore (1999). The experience economy, work is theatre and every business a stage. Harvard Business School Press, Boston.

Ramírez, M. S. y Mortera, F. J. (2009). Implementación y Desarrollo del Portal Académico de Recursos Educativos Abiertos (REAs): Knowledge Hub para Educación Básica. Memorias del IV Congreso Nacional de Posgrados en Educación. Guanajuato, México. Recuperado el 28 de junio de 2015, de http://bit.ly/1JgVzXE.

Regil-Vargas, Laura (2003). Interactividad: Construcción de la Mirada. Recuperado el 21 de junio de 2015, de http://bit.ly/1BFucXl.

Regil-Vargas, Laura (2014). Cultura digital universitaria. Tesis doctoral. Universidad Autónoma de Barcelona. Facultad de Ciencias de la Comunicación.

Rodríguez Rivera, Ma. Antonieta (2014). El Diseño de Interfaz gráfica para cursos en línea. Recuperado el 21 de junio de 2015, de http://bit.ly/1LnoFHY.

Universidad Nacional Autónoma de México. (13 agosto 2015). Red Universitaria de Aprendizaje. Recuperado el 23 de agosto de 2015, de http://www.rua.unam.mx/

Warger, Thomas (2002). The Open-Source Movement. Edutech International. Recuperado el 25 de junio de 2015, de http://bit.ly/1GuD5j3. 